\title{
Bode Integral Constraints, Colocation, and Spillover in Active Noise and Vibration Control
}

\author{
Jeongho Hong and Dennis S. Bernstein
}

\begin{abstract}
With the success of feedforward techniques for active noise control, feedback control researchers have begun to explore the relationship between these two control paradigms. The goal of this paper is to further investigate this relationship by means of the classical Bode integral constraint on achievable performance. This constraint provides insight into the phenomenon of spillover which we define as disturbance amplification by the closedloop system relative to the open-loop transfer function gain. Specifically, it is shown that a particular feedforward controller called the zero spillover controller avoids spillover by producing perfect disturbance cancellation at every frequency. The analysis suggests that spillover can be avoided only if the control speaker and the disturbance source are noncolocated and the performance microphone and the measurement microphone are noncolocated. For realizability, we derive the approximate zero spillover controller which is shown to be an optimal feedback controller for an LQG problem with suitable cross weighting. Finally, the results are illustrated by means of structural and acoustic examples.
\end{abstract}

Index Terms-Bode integral constraint, colocation, LQG, positive real, spillover.

\section{INTRODUCTION}

A LTHOUGH feedforward control techniques are widely used in active noise control applications [1], an alternative approach is to apply standard feedback control techniques. Although feedback techniques have seen relatively limited use in noise control problems, there have been an increasing number of applications. For example, the feasibility of feedback control for suppressing noise in a one-dimensional duct was shown in [2], while, in related experiments [3], [4], modelbased feedback controllers were used to obtain broad-band noise suppression.

With the success of feedforward techniques, feedback control researchers have begun to explore the relationship between these two control paradigms [5]. The goal of the present paper is to further investigate this relationship by means of the classical Bode integral constraint on sensitivity [6]-[9]. Specifically, we show that the geometric arrangement of speakers and microphones in the standard feedforward setup allows the control designer to effectively circumvent the inherent performance limitations of the Bode integral, which, for a stable loop transfer function $L(s)$ with relative degree at least 2 , constrains the integrated log-magnitude of the sensitivity function $S(s)$ to be zero, where $S(s)=1 /[1-$ $L(s)]$.

Manuscript received September 6, 1995; revised September 19, 1996 Recommended by Associate Editor, E. G. Collins, Jr. This work was supported in part by the Air Force Office of Scientific Research under Grant F4962095-1-0019.

The authors are with the Department of Aerospace Engineering, The University of Michigan, Ann Arbor, MI 48109-2140 USA.

Publisher Item Identifier S 1063-6536(98)00581-8.
The basis of our work is the fact that in the singlesensor/single-actuator case, the closed-loop transfer function can be written as the product of the sensitivity function and a spatial component called the spillover function, which depends upon the physical arrangement of the measurement, control, disturbance and performance. It turns out that if the control speaker and the disturbance source are noncolocated and if the performance microphone and the measurement microphone are noncolocated, then it is possible to overcome the Bode constraint in the sense that arbitrary attenuation of the open-loop transfer function can be achieved. In fact, control strategies for minimizing the spillover function appear in the feedforward literature for broadband suppression. A controller that exploits this structure was given in [10] and is described in [11, p. 186]. This controller, which we call the zero spillover controller, was obtained as a singular multiloop LQG controller in [12].

In addition to clarifying the distinctions and similarities between traditional feedback control techniques and more specialized feedforward noise control methods, the Bode constraint helps to clarify the phenomenon of spillover which is widely discussed in the feedback vibration control literature [13], [14]. In [13], control spillover is defined to be the excitation by the actuator of unmodeled plant dynamics, while observation spillover is defined to be the sensing by the sensor of unmodeled plant dynamics. In the present paper, we say that spillover occurs at frequency $\omega$ if the closedloop transfer function magnitude $\left|\tilde{G}_{z_{x}} w(\chi \omega)\right|$ is greater than the open-loop transfer function magnitude $\left|G_{z_{x}} w(\not \omega)\right|$. With this definition, spillover can occur whether or not the plant possesses unmodeled dynamics.

In this paper, the Bode constraint is used to identify situations in which spillover is unavoidable regardless of the linear time-invariant control law (assuming relative degree 2 or greater loop transfer function). In particular, our results show that spillover can be avoided only if 1) the control speaker and the disturbance source are noncolocated and 2) the performance microphone and the measurement microphone are noncolocated. Note that these conditions can be satisfied whether or not the control speaker and the measurement microphone are colocated. In fact, whether or not the control speaker and the measurement microphone are colocated has greater impact on robust stability than on achievable nominal performance. In fact, it follows from singular LQG theory that arbitrarily good nominal performance can be achieved if and only if the transfer functions from the control speaker to the performance microphone and from the disturbance source to the measurement microphone are both minimum phase [15]. Note that this result does not require that the transfer function 
from the control speaker to the measurement microphone be minimum phase, in particular, colocated. ${ }^{1}$

Finally, the consequences of colocation in vibration and noise control are discussed in [16]-[19].

\section{PRELIMINARIES}

Consider a control problem represented by a linear timeinvariant system with transfer matrix $G(s)$ partitioned as

$$
G(s)=\left[\begin{array}{ll}
G_{z_{x} w}(s) & G_{z_{x} u}(s) \\
G_{y_{x} w}(s) & G_{y_{x} u}(s)
\end{array}\right]
$$

with realization

$$
G(s) \sim\left[\begin{array}{c|cc}
A & D_{1} & B \\
\hline E_{1} & 0 & 0 \\
C & 0 & 0
\end{array}\right] .
$$

The state performance variable $z_{x}$, noiseless measurement $y_{x}$, disturbance signal $w$ and control input $u$ are related by

$$
\begin{aligned}
& z_{x}=G_{z_{x} w}(s) w+G_{z_{x} u}(s) u \\
& y_{x}=G_{y_{x} w}(s) w+G_{y_{x} u}(s) u
\end{aligned}
$$

and satisfy the state-space equations

$$
\begin{aligned}
\dot{x}(t) & =A x(t)+B u(t)+D_{1} w(t) \\
z_{x}(t) & =E_{1} x(t) \\
y_{x}(t) & =C x(t)
\end{aligned}
$$

where $x(t) \in \Re^{n}$. The control input $u$ is given by

$$
u=G_{\mathrm{C}}(s) y_{x}
$$

where $G_{\mathrm{C}}(s)$ is the controller transfer function. We say that the noiseless measurement $y_{x}$ and state performance variable $z_{x}$ are colocated if $C=E_{1}$ [so that $z_{x}(t)=y_{x}(t)$ ], while the disturbance signal $w$ and the control input $u$ are colocated if $B=D_{1}$.

Assuming that $z_{x}, y_{x}, w$, and $u$ are scalars, the closed-loop transfer function from $w$ to $z_{x}$ can be written as

$$
\tilde{G}_{z_{x} w}(s)=F\left[G_{\mathrm{C}}(s)\right] S(s)
$$

where

$$
\begin{aligned}
F\left[G_{\mathrm{c}}(s)\right] \triangleq & G_{z_{x} w}(s)-\left[G_{z_{x} w}(s) G_{y_{x} u}(s)\right. \\
& \left.-G_{z_{x} u}(s) G_{y_{x} w}(s)\right] G_{\mathrm{c}}(s)
\end{aligned}
$$

and

$$
S(s) \triangleq \frac{1}{1-L(s)}, \quad L(s) \triangleq G_{y_{x} u}(s) G_{\mathrm{c}}(s)
$$

Note that $\tilde{G}_{z_{x} w}(s)$ is the product of two terms, namely, the spillover function $F\left[G_{\mathrm{C}}(s)\right]$, which depends upon the physical arrangement of the measurement, control, disturbance, and performance, and the sensitivity function $S(s)$, which depends upon the loop transfer function $L(s)$.

\footnotetext{
${ }^{1}$ Recall that a colocated (driving point) admittance transfer function from force to velocity, from voltage to current, or from pressure to volume velocity is dual, that is, the product of the input and output signals has the dimensions of power. Such a transfer function is positive real and thus minimum phase.
}

\section{Bode Sensitivity InTEGRAL CONSTRAINT AND SPILLOVER}

For the special case in which the loop transfer function $L(s)$ is asymptotically stable and has relative degree at least two, the Bode sensitivity integral constraint [6]-[8] states that

$$
\int_{0}^{\infty} \log |S(\jmath \omega)| d \omega=0 .
$$

An immediate consequence of (12) is that the magnitude $|S(\chi \omega)|$ of the sensitivity function cannot be less than unity at every frequency.

Now consider the case in which the state performance variable $z_{x}$ and the noiseless measurement $y_{x}$ are colocated so that $G_{z_{x} w}(s)=G_{y_{x} w}(s)$ and $G_{z_{x} u}(s)=G_{y_{x} u}(s)$ and the case in which the disturbance signal $w$ and the control input $u$ are colocated so that $G_{z_{x} w}(s)=G_{z_{x} u}(s)$ and $G_{y_{x} w}(s)=$ $G_{y_{x} u}(s)$. In both of these cases it follows from (10) that the spillover function satisfies

$$
F\left[G_{c}(\jmath \omega)\right]=G_{z_{x} w}(\ldots \omega)
$$

and thus, by (9)

$$
\tilde{G}_{z_{x} w}(\jmath \omega)=G_{z_{x} w}(\jmath \omega) S(\jmath \omega)
$$

so that

$$
\left|\tilde{G}_{z_{x} w}(\jmath \omega)\right|=\left|G_{z_{x} w}(\jmath \omega)\right||S(\not \omega)| .
$$

Thus, in either case, since the magnitude $|S(\jmath \omega)|$ of the sensitivity function cannot be less than unity at all frequencies, it follows that $\left|\tilde{G}_{z_{x} w}(\not \omega)\right|$ must be greater than $\left|G_{z_{x} w}(. \jmath)\right|$ at some frequencies. This phenomenon, namely, that $\left|G_{z_{x}} w(\jmath \omega)\right|$ is greater than $\left|G_{z_{x}} w(\jmath \omega)\right|$ at a frequency $\omega$, is defined as spillover. It is important to note that in the cases discussed above, spillover (as defined here) is unavoidable even if the system model $G(s)$ is a completely accurate representation of the physical system, that is, whether or not the plant has parameter uncertainty or unmodeled dynamics. In theory, the effect of spillover can be reduced by choosing controllers so that $|S(\not \omega)| \approx 1$ over a wide frequency range [20]. In practice, however, the ability to do this is subject to bandwidth and saturation limitations of the control actuator and electronics.

\section{ZERO SPILlOVER CONTROLleR AND FEEDFORWARD CONTROL}

It was shown in Section III that if the state performance variable and noiseless measurement are colocated or the disturbance signal and control input are colocated, then spillover cannot be avoided. We thus consider the case in which the state performance variable and noiseless measurement are noncolocated and the disturbance signal and control input are noncolocated. Clearly, spillover is avoided in the case of perfect cancellation, that is, when $\tilde{G}_{z_{x} w}(s)=0$, which implies that the spillover function satisfies $F\left[G_{\mathrm{C}}(s)\right]=0$. The resulting zero spillover controller is given by

$$
G_{\mathrm{ZSC}}(s)=\frac{G_{z_{x} w}(s)}{G_{z_{x} w}(s) G_{y_{x} u}(s)-G_{z_{x} u}(s) G_{y_{x} w}(s)} .
$$


Since $G_{\mathrm{ZSC}}(s)$ yields $F\left[G_{\mathrm{ZSC}}(s)\right]=0$, it follows from (9) that $\tilde{G}_{z_{x} w}(s)=0$ and thus $G_{\mathrm{ZSC}}(s)$ provides perfect cancellation of the disturbance at every frequency and hence produces no spillover. This property of $G_{\mathrm{ZSC}}(s)$ was noted in [12]. We shall say that the zero spillover controller $G_{\mathrm{ZSC}}(s)$ exists if the denominator of $G_{\mathrm{ZSC}}(s)$ in (16) is not identically equal to zero.

Note that the expression (16) for $G_{\mathrm{ZSC}}(s)$ indicates plant inversion and thus possible sensitivity to plant uncertainty. In addition, if either the state performance variable and noiseless measurement are colocated so that $G_{z_{x} w}(s)=G_{y_{x} w}(s)$ and $G_{z_{x} u}(s)=G_{y_{x} u}(s)$, or the disturbance source and the control input are colocated so that $G_{z_{x} w}(s)=G_{z_{x} u}(s)$ and $G_{y_{x} w}(s)=G_{y_{x} u}(s)$, then the denominator of $G_{\mathrm{ZSC}}(s)$ is identically equal to zero, and thus $G_{\mathrm{ZSC}}(s)$ does not exist. However, these cases do not occur in feedforward control where the measurement microphone is usually placed near the disturbance source and far from the performance location, while the disturbance occurs far from the control speaker which is typically located downstream in a duct.

It can be shown that the zero spillover controller $G_{\mathrm{ZSC}}(s)$ may be improper. Thus the loop transfer function $L(s)=$ $G_{y_{x} u}(s) G_{\mathrm{ZSC}}(s)$ may have relative degree less than two and hence the Bode integral constraint (12) may not be satisfied. However, the zero spillover controller yields $\tilde{G}_{z_{x} w}(s)=$ 0 whether or not (12) applies. The following result gives conditions under which $G_{\mathrm{ZSC}}(s)$ stabilizes the sensitivity function $S(s)$.

Proposition 4.1: Suppose $G(s)$ is asymptotically stable, $G_{z_{x} u}(s)$ and $G_{y_{x} w}(s)$ are minimum phase, and $G_{\mathrm{ZSC}}(s)$ exists. Then the sensitivity function $S(s)$ is asymptotically stable.

Proof: Letting num[·] and den[·] denote numerator and denominator, respectively, it follows that:

$$
\begin{aligned}
\operatorname{den}[S(s)]= & \operatorname{num}\left[1-G_{\mathrm{ZSC}}(s) G_{y_{x} u}(s)\right] \\
= & \operatorname{num}\left[1-\frac{G_{z_{x} w}(s) G_{y_{x} u}(s)}{G_{z_{x} w}(s) G_{y_{x} u}(s)-G_{z_{x} u}(s) G_{y_{x} w}(s)}\right] \\
= & \operatorname{num}\left[G_{z_{x} u}(s)\right] \operatorname{num}\left[G_{y_{x} w}(s)\right] \\
& \cdot \operatorname{den}\left[G_{z_{x}}(s)\right] \operatorname{den}\left[G_{y_{x} u}(s)\right] .
\end{aligned}
$$

Since the open-loop system is asymptotically stable and since the transfer functions $G_{z_{x} u}(s)$ and $G_{y_{x} w}(s)$ are minimum phase, it follows that $\operatorname{den}[S(s)]$ is Hurwitz.

Next we show that the zero spillover controller (16) is identical to the feedforward controller given in [10]. In the notation of [10], Roure considers the controller

$$
C(f)=\frac{H_{0}(f)}{H_{1}(f) H_{0}(f)-H_{2}(f)}
$$

where $f=\omega / 2 \pi, H_{1}(f)$ is the transfer function from the control speaker to the measurement microphone, $H_{2}(f)$ is the transfer function from the control speaker to the performance microphone, and $H_{0}(f)$ is the transfer function from the measurement microphone to the performance microphone. In the notation of (1), the appropriate correspondences are

$$
\begin{aligned}
& H_{1}(f)=G_{y_{x} u}(\jmath \omega), \quad H_{2}(f)=G_{z_{x} u}(\jmath \omega), \\
& H_{0}(f)=\frac{G_{z_{x} w}(\jmath \omega)}{G_{y_{x} w}(\jmath \omega)} .
\end{aligned}
$$

Thus

$$
\begin{aligned}
C(f) & =\frac{\frac{G_{z_{x} w}(\jmath \omega)}{G_{y_{x} w}(\jmath \omega)}}{G_{y_{x} u}(\jmath \omega) \frac{G_{z_{x}} w(\jmath \omega)}{G_{y_{x} w}(\jmath \omega)}-G_{z_{x} u}(\jmath \omega)} \\
& =\frac{G_{z_{x} w}(\jmath \omega)}{G_{z_{x} w}(\jmath \omega) G_{y_{x} u}(\jmath \omega)-G_{z_{x} u}(\jmath \omega) G_{y_{x} w}(\jmath \omega)} \\
& =G_{\mathrm{ZSC}}(\jmath \omega) .
\end{aligned}
$$

The ability of the zero spillover controller to avoid spillover can be seen in [10, Fig. 5] where significant noise reduction with minimal spillover was achieved over a broad frequency band. If $G_{\mathrm{ZSC}}(s)$ is improper, then it is not physically realizable. Therefore, in the following section we consider a strictly proper modification of the zero spillover controller.

\section{APProximate Zero SPILlover CONTROLler}

In this section, we continue to assume that $z, y, u$, and $w$ are scalar and we derive a state-space realization for a modification of the zero spillover controller. To do this, we introduce a nonzero control weighting $E_{2}$ and nonzero measurement noise $D_{2} w$ such that (6) and (7) are replaced by

$$
\begin{aligned}
& z(t)=E_{1} x(t)+E_{2} u(t) \\
& y(t)=C x(t)+D_{2} w(t) .
\end{aligned}
$$

The modified version of the zero spillover controller (16) corresponding to (5), (19), and (20) will be called the approximate zero spillover controller $G_{\mathrm{AZSC}}(s)$ and is given by the strictly proper transfer function

$$
G_{\mathrm{AZSC}}(s)=\frac{G_{z w}(s)}{G_{z w}(s) G_{y u}(s)-G_{z u}(s) G_{y w}(s)} .
$$

Comparing (16) and (21) suggests that the zero spillover controller can be recovered from the approximate zero spillover controller by letting $E_{2} \rightarrow 0$ and $D_{2} \rightarrow 0$, that is

$$
\lim _{E_{2}, D_{2} \rightarrow 0} G_{\mathrm{AZSC}}(s)=G_{\mathrm{ZSC}}(s) .
$$

However, $G_{\mathrm{ZSC}}(s)$ may not exist and thus (22) may not be valid.

We now obtain a state-space realization for the approximate zero spillover controller. Unlike the zero spillover controller, this development does not exclude the colocated cases $C=E_{1}$ and $B=D_{1}$. With (19) and (20), we redefine the system (1) as

$$
G(s)=\left[\begin{array}{ll}
G_{z w}(s) & G_{z u}(s) \\
G_{y w}(s) & G_{y u}(s)
\end{array}\right]
$$


with the state-space realizations

$$
\begin{array}{ll}
G_{z w}(s) \sim\left[\begin{array}{c|c}
A & D_{1} \\
\hline E_{1} & 0
\end{array}\right], \quad G_{z u}(s) \sim\left[\begin{array}{c|c}
A & B \\
\hline E_{1} & E_{2}
\end{array}\right] \\
G_{y w}(s) \sim\left[\begin{array}{l|c}
A & D_{1} \\
\hline C & D_{2}
\end{array}\right], \quad G_{y u}(s) \sim\left[\begin{array}{c|c}
A & B \\
\hline C & 0
\end{array}\right] .
\end{array}
$$

By applying formulas for parallel and cascade interconnections of transfer functions and for the inverse of a transfer function to (21), it follows that $G_{\mathrm{AZSC}}(s)$ has a realization of order $5 n$ given by (24) at the bottom of the page. However, this realization is not minimal and its order can be reduced by applying the state transformation matrix

$$
\begin{aligned}
& S_{1}= \\
& {\left[\begin{array}{ccccc}
0 & 0 & 0 & 0 & I \\
0 & 0 & D_{1}^{\mathrm{T}} D_{1} I & 0 & -B D_{1}^{\mathrm{T}} \\
0 & D_{1}^{\mathrm{T}} D_{1} I & D_{1}^{\mathrm{T}} D_{1} D_{2} I & -D_{1}^{\mathrm{T}} D_{1} I & 0 \\
I & 0 & 0 & 0 & -I \\
0 & 0 & 0 & I & 0
\end{array}\right]} \\
& S_{1}^{-1}= \\
& {\left[\begin{array}{cccccc}
-B D_{1}^{\mathrm{T}} D_{2} / D_{1}^{\mathrm{T}} D_{1} & -D_{2} I / D_{1}^{\mathrm{T}} D_{1} & I / D_{1}^{\mathrm{T}} D_{1} & 0 & I \\
B D_{1}^{\mathrm{T}} / D_{1}^{\mathrm{T}} D_{1} & I / D_{1}^{\mathrm{T}} D_{1} & 0 & 0 & 0 \\
0 & 0 & 0 & 0 & I \\
I & 0 & 0 & 0 & 0
\end{array}\right] .}
\end{aligned}
$$

The transformed state $S_{1} x$ has $n$ uncontrollable states and $n$ unobservable states which can be truncated to yield the order $3 n$ realization given in (26) at the bottom of the page. If $B=D_{1}$, then by applying the state transformation matrix

$$
\begin{aligned}
& S_{2}=\left[\begin{array}{ccc}
B D_{1}^{\mathrm{T}} / D_{1}^{\mathrm{T}} D_{1} & I / D_{1}^{\mathrm{T}} D_{1} & 0 \\
0 & 0 & I / D_{1}^{\mathrm{T}} D_{1} D_{2} \\
I-B D_{1}^{\mathrm{T}} / D_{1}^{\mathrm{T}} D_{1} & -I / D_{1}^{\mathrm{T}} D_{1} & 0
\end{array}\right] \\
& S_{2}^{-1}=\left[\begin{array}{ccc}
I & 0 & I \\
-B D_{1}^{\mathrm{T}}+D_{1}^{\mathrm{T}} D_{1} I & 0 & -B D_{1}^{\mathrm{T}} \\
0 & D_{1}^{\mathrm{T}} D_{1} D_{2} I & 0
\end{array}\right]
\end{aligned}
$$

the transformed state $S_{2} x$ has $2 n$ uncontrollable states which can be truncated to yield the order $n$ realization

$$
G_{\mathrm{AZSC}}(s) \sim\left[\begin{array}{c|c}
A-B E_{1} / E_{2}-D_{1} C / D_{2} & D_{1} / D_{2} \\
\hline-E_{1} / E_{2} & 0
\end{array}\right] .
$$

Numerical evidence strongly suggests that (27) is valid in general, even if $D_{1} \neq B$.

It now remains to determine conditions under which the approximate zero spillover controller stabilizes the closed-loop transfer function. This question, along with connections to LQG control, is addressed in the following section.

\section{LQG CONTROL AND a Multivariable Generalization OF THE APPROXIMATE ZERO SPILLOVER CONTROLLER}

The realization (28) of the approximate zero spillover controller was obtained for the case in which $z, y, u$, and $w$ are scalar. In this section, we generalize the approximate zero spillover controller to the multivariable case by using LQG control. Consider the $\mathrm{H}_{2}$ norm of the closed-loop transfer function $\tilde{G}_{z w}(s)$ given by

$$
J\left(G_{\mathrm{c}}\right)=\left\|\tilde{G}_{z w}\right\|_{2}^{2}
$$

where the controller $G_{\mathrm{C}}(s)$ has a realization

$$
G_{\mathrm{C}}(s) \sim\left[\begin{array}{c|c}
A_{\mathrm{c}} & B_{\mathrm{c}} \\
\hline C_{\mathrm{C}} & 0
\end{array}\right] .
$$

The following result yields the $\mathrm{H}_{2}$-optimal controller for the case in which $\operatorname{dim} z=\operatorname{dim} u$ and $\operatorname{dim} y=\operatorname{dim} w$. In this case, $E_{2}$ and $D_{2}$ are square matrices.

Theorem 6.1: Suppose that $E_{2}$ and $D_{2}$ are invertible and that $A-B E_{2}^{-1} E_{1}$ and $A-D_{1} D_{2}^{-1} C$ are asymptotically stable. Then the $\mathrm{H}_{2}$-optimal controller is given by

$$
G_{\mathrm{c}, \mathrm{opt}}(s) \sim\left[\begin{array}{c|c}
A-B E_{2}^{-1} E_{1}-D_{1} D_{2}^{-1} C & D_{1} D_{2}^{-1} \\
\hline-E_{2}^{-1} E_{1} & 0
\end{array}\right] .
$$

Furthermore

$$
J\left(G_{\mathrm{c}, \mathrm{opt}}\right)=0
$$

$$
\begin{aligned}
& G_{\mathrm{AZSC}}(s) \sim \\
& \left.\qquad \begin{array}{ccc|c}
A-D_{1} C / D_{2}-D_{1} E_{1} B D_{1}^{\mathrm{T}} /\left(E_{2} D_{1}^{\mathrm{T}} D_{1}\right) & -D_{1} E_{1} /\left(D_{1}^{\mathrm{T}} D_{1} E_{2}\right) & D_{1} E_{1} /\left(D_{1}^{\mathrm{T}} D_{1} D_{2} E_{2}\right) & D_{1} / D_{2} \\
A B D_{1}^{\mathrm{T}}-B D_{1}^{\mathrm{T}} A & A & 0 & 0 \\
D_{1} C B D_{1}^{\mathrm{T}}-B D_{1}^{\mathrm{T}} D_{1} C & D_{1} C & A & 0 \\
\hline-E_{1} / E_{2} & 0 & 0 & 0
\end{array}\right]
\end{aligned}
$$


and thus $\tilde{G}_{z w}(s)=0$. Finally, the sensitivity function $S(s)$ is asymptotically stable.

Proof: The $\mathrm{H}_{2}$ regulator Riccati equation with nonzero cross weighting has the form

$$
\begin{aligned}
0= & \left(A-B R_{2}^{-1} R_{12}^{\mathrm{T}}\right)^{\mathrm{T}} P+P\left(A-B R_{2}^{-1} R_{12}^{\mathrm{T}}\right) \\
& -P B R_{2}^{-1} B^{\mathrm{T}} P+R_{1}-R_{12} R_{2}^{-1} R_{12}^{\mathrm{T}}
\end{aligned}
$$

where $R_{1}=E_{1}^{\mathrm{T}} E_{1}, R_{12}=E_{1}^{\mathrm{T}} E_{2}$ and $R_{2}=E_{2}^{\mathrm{T}} E_{2}$. Since $E_{2}$ and $D_{2}$ are invertible, it follows that $R_{12} R_{2}^{-1} R_{12}^{\mathrm{T}}=$ $E_{1}^{\mathrm{T}} E_{2}\left(E_{2}^{\mathrm{T}} E_{2}\right)^{-1} E_{2}^{\mathrm{T}} E_{1}=E_{1}^{\mathrm{T}} E_{1}=R_{1}$ and $R_{2}^{-1} R_{12}^{\mathrm{T}}=$ $\left(E_{2}^{\mathrm{T}} E_{2}\right)^{-1}\left(E_{1}^{\mathrm{T}} E_{2}\right)^{\mathrm{T}}=E_{2}^{-1} E_{1}$. Thus, the regulator Riccati equation becomes

$0=\left(A-B E_{2}^{-1} E_{1}\right)^{\mathrm{T}} P+P\left(A-B E_{2}^{-1} E_{1}\right)-P B R_{2}^{-1} B^{\mathrm{T}} P$

Since $A-B E_{2}^{-1} E_{1}$ is asymptotically stable, the unique stabilizing solution to this Riccati equation is ${ }^{2} P=0$. Likewise, for the observer Riccati equation with correlated plant disturbance and measurement noise given by

$$
\begin{aligned}
0= & \left(A-V_{12} V_{2}^{-1} C\right) Q+Q\left(A-V_{12} V_{2}^{-1} C\right)^{\mathrm{T}} \\
& -Q C^{\mathrm{T}} V_{2}^{-1} C Q+V_{1}-V_{12} V_{2}^{-1} V_{12}^{\mathrm{T}}
\end{aligned}
$$

where $V_{1}=D_{1} D_{1}^{\mathrm{T}}, V_{12}=D_{1} D_{2}^{\mathrm{T}}$, and $V_{2}=D_{2} D_{2}^{\mathrm{T}}$, the unique stabilizing solution is $Q=0$. With these solutions, the $\mathrm{H}_{2}$-optimal controller has the form (31). Furthermore, since $Q=P=0$, the $\mathrm{H}_{2}$-optimal cost is given by

$$
\begin{aligned}
J\left(G_{\text {c,opt }}\right)= & \operatorname{tr}\left[Q R_{1}+P\left(V_{12}+Q C^{\mathrm{T}}\right)\right. \\
& \left.\cdot V_{2}^{-1}\left(V_{12}+Q C^{\mathrm{T}}\right)^{\mathrm{T}}\right]=0 .
\end{aligned}
$$

Thus, it follows from (29) that ${ }^{3} \tilde{G}_{z w}(s)=0$.

With the controller (31), the sensitivity function $S(s)$ has the realization

$$
S(s) \sim\left[\begin{array}{cc|c}
A & B C_{\mathrm{c}} & B \\
B_{\mathrm{C}} C & A_{\mathrm{c}} & 0 \\
\hline 0 & C_{\mathrm{c}} & 1
\end{array}\right]
$$

where $A_{\mathrm{c}}=A+B C_{\mathrm{c}}-B_{\mathrm{c}} C, B_{\mathrm{c}}=D_{1} D_{2}^{-1}$, and $C_{\mathrm{c}}=$ $-E_{2}^{-1} E_{1}$. Under the assumptions of Theorem 6.1 and with the controller (31), it follows that:

$$
\begin{aligned}
& {\left[\begin{array}{cc}
A & B C_{\mathrm{c}} \\
B_{\mathrm{c}} C & A_{\mathrm{c}}
\end{array}\right]=} \\
& {\left[\begin{array}{ll}
I & 0 \\
I & I
\end{array}\right]\left[\begin{array}{cc}
A+B C_{\mathrm{c}} & B C_{\mathrm{C}} \\
0 & A-B_{\mathrm{c}} C
\end{array}\right]\left[\begin{array}{ll}
I & 0 \\
I & I
\end{array}\right]^{-1}}
\end{aligned}
$$

where

$$
\begin{aligned}
& A+B C_{\mathrm{c}}=A-B E_{2}^{-1} E_{1} \\
& A-B_{\mathrm{c}} C=A-D_{1} D_{2}^{-1} C .
\end{aligned}
$$

Hence $S(s)$ is asymptotically stable.

\footnotetext{
${ }^{2}$ Since $P=0$ is the stabilizing solution, it is also the maximal solution (see [21]). Therefore, it is the only nonnegative-definite solution.

${ }^{3}$ This can be confirmed directly as well.
}

Theorem 6.1 provides a specialized LQG controller that involves cross weightings. To interpret (32), write the $\mathrm{H}_{2}$ cost as

$$
\begin{aligned}
J\left(G_{c}\right)= & \lim _{t \rightarrow \infty} \frac{1}{t} \mathbb{E}\left\{\int _ { 0 } ^ { t } \left[x^{\mathrm{T}}(s) E_{1}^{\mathrm{T}} E_{1} x(s)\right.\right. \\
& \left.\left.+2 x^{\mathrm{T}}(s) E_{1}^{\mathrm{T}} E_{2} u(s)+u^{\mathrm{T}}(s) E_{2}^{\mathrm{T}} E_{2} u(s)\right] d s\right\}
\end{aligned}
$$

where $w(t)$ in (5) and (20) is white noise with unit intensity. The optimal cost (32) shows that the cross-weighting term $x^{\mathrm{T}} E_{1}^{\mathrm{T}} E_{2} u$ is negative and exactly cancels the state and control terms $x^{\mathrm{T}} E_{1}^{\mathrm{T}} E_{1} x$ and $u^{\mathrm{T}} E_{2}^{\mathrm{T}} E_{2} u$.

Note that the $\mathrm{H}_{2}$-optimal controller (31) and the approximate zero spillover controller (28) are identical, that is

$$
G_{\mathrm{AZSC}}(s)=G_{\mathrm{c}, \mathrm{opt}}(s)
$$

in the case in which $z, y, u$, and $w$ are scalar and under the assumption that (28) is equivalent to (26), The LQG controller $G_{c \text {, opt }}(s)$ thus provides a multivariable generalization of the approximate zero spillover controller.

If the transfer functions from the control input $u$ to the state performance variable $z_{x}$ and from the disturbance signal $w$ to the noiseless measurement $y_{x}$ are positive real, then $A-B E_{2}^{-1} E_{1}$ and $A-D_{1} D_{2}^{-1} C$ are asymptotically stable for all $E_{2}$ and $D_{2}$ such that $E_{2}+E_{2}^{\mathrm{T}}$ and $D_{2}+D_{2}^{\mathrm{T}}$ are positive definite. This result follows from the fact that the negative feedback interconnection of a positive real transfer function and a strictly positive real transfer function is asymptotically stable [22]. Furthermore, note that the transfer functions from the control input $u$ to the state performance variable $z_{x}$ and from the disturbance signal $w$ to the noiseless measurement $y_{x}$ are positive real if the control input $u$ and the state performance variable $z_{x}$ are colocated and dual and the disturbance signal $w$ and the noiseless measurement $y_{x}$ are colocated and dual. These positive real conditions guarantee stability as $E_{2}, D_{2} \rightarrow$ 0 in recovering $G_{\mathrm{ZSC}}(s)$ from $G_{\mathrm{AZSC}}(s)$ as in (22). Note that this spatial arrangement does not violate the noncolocation requirements for avoiding spillover discussed in Section III. A summary of the various cases is given in Table I. Finally, note that Proposition 4.1 shows that $G_{\mathrm{ZXU}}(s)$ and $G_{\mathrm{YXW}}(s)$ need only be minimum phase in order to guarantee that the sensitivity function $S(s)$ is asymptotically stable for the controller $G_{\mathrm{ZSC}}(s)$

\section{EXAMPLES}

\section{A. Two-Mass Example}

The equations of motion for the two-mass system shown in Fig. 1 are given by

$$
\begin{gathered}
m_{1} \ddot{q}_{1}+c_{1} \dot{q}_{1}+k_{1} q_{1}+c_{2}\left(\dot{q}_{1}-\dot{q}_{2}\right)+k_{2}\left(q_{1}-q_{2}\right)=w \\
m_{2} \ddot{q}_{2}+c_{2}\left(\dot{q}_{2}-\dot{q}_{1}\right)+k_{2}\left(q_{2}-q_{1}\right)+c_{3} \dot{q}_{2}+k_{2} q_{2}=u \\
z_{x}=\dot{q}_{2}, \quad y_{x}=\dot{q}_{1}
\end{gathered}
$$


TABLE I

CONSEQUENCES OF COLOCATION

\begin{tabular}{|l|l|}
\hline 1$) z_{x}$ and $y_{x}$ are colocated. & $\begin{array}{l}\text { Spillover is unavoidable } \\
\text { [see (15)]. }\end{array}$ \\
\hline 2) $w$ and $u$ are colocated. & $\begin{array}{l}\text { Spillover is unavoidable } \\
\text { [see (15)]. }\end{array}$ \\
\hline $\begin{array}{l}\text { 3). } G(s) \text { is asymptotically stable } \\
\text { and } G_{z_{x} u}(s) \text { and } G_{y_{x} w}(s) \text { are } \\
\text { minimum phase. }\end{array}$ & $\begin{array}{l}\text { Arbitrarily good nominal } \\
\text { performance is possible [15]. If } \\
G_{\text {ZSC }}(s) \text { exists, then it stabilizes } \\
S(s) \text { and } \tilde{G}\end{array} z_{x} w$ \\
\hline $\begin{array}{l}\text { 4) } G_{z_{x} u}(s) \text { and } G_{y_{x} w}(s) \text { are } \\
\text { positive real. }\end{array}$ & $\begin{array}{l}\text { Arbitrarily good nominal } \\
\text { performance is possible using } \\
G_{\text {AzsC }}(s), \text { which is proper and } \\
\text { stabilizing. }\end{array}$ \\
\hline 5) $G_{y_{x} u}(s)$ is positive real. & $\begin{array}{l}\text { Robust stability is possible via } \\
\text { dissipative control [23], [24]. }\end{array}$ \\
\hline
\end{tabular}

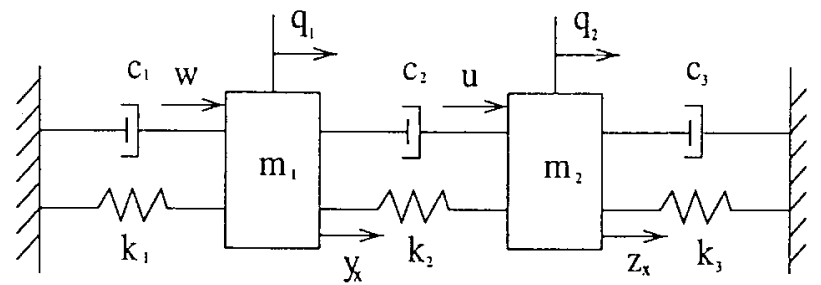

Fig. 1. Two-mass system.

which yields the system shown in (38) shown at the bottom of the page, and the zero spillover controller

$$
G_{\mathrm{ZSC}}(s)=-c_{2}-\frac{k_{2}}{s}
$$

With this PI controller, the loop transfer function and sensitivity are given by (39) shown at the bottom of the page. It can be verified directly that $F\left[G_{\mathrm{ZSC}}(s)\right]=0$ and thus $\tilde{G}_{z_{x} w}(s)=0$ so that perfect disturbance rejection has been achieved despite the fact that the Bode constraint (12) on the sensitivity is satisfied. It can be seen that the sensitivity function $S(s)$ is asymptotically stable, which is consistent with Proposition 4.1.

To obtain the approximate zero spillover controller, let $x_{1}=q_{1}, x_{2}=\dot{q}_{1}, x_{3}=q_{2}, x_{4}=\dot{q}_{2}$ and consider the state-space description

$$
\begin{aligned}
& \dot{x}=\left[\begin{array}{cccc}
0 & 1 & 0 & 0 \\
-\frac{k_{1}+k_{2}}{m_{1}} & -\frac{c_{1}+c_{2}}{m_{1}} & \frac{k_{2}}{m_{1}} & \frac{c_{2}}{m_{1}} \\
0 & 0 & 0 & 1 \\
\frac{k_{2}}{m_{2}} & \frac{c_{2}}{m_{2}} & -\frac{k_{2}+k_{3}}{m_{2}} & -\frac{c_{2}+c_{3}}{m_{2}}
\end{array}\right] x \\
& +\left[\begin{array}{c}
0 \\
0 \\
0 \\
\frac{1}{m_{2}}
\end{array}\right] u+\left[\begin{array}{c}
0 \\
\frac{1}{m_{1}} \\
0 \\
0
\end{array}\right] w \\
& y=\left[\begin{array}{llll}
0 & 1 & 0 & 0
\end{array}\right] x+D_{2} w \\
& z=\left[\begin{array}{llll}
0 & 0 & 0 & 1
\end{array}\right] x+E_{2} u \text {. }
\end{aligned}
$$

It now follows from (27) that $G_{\mathrm{AZSC}}(s)$ is given by (40) at the bottom of the next page, where

$$
\begin{aligned}
g(s)= & \frac{1}{s^{2}}\left(D_{2} E_{2} m_{1} m_{2} s^{4}+\left\{D_{2} m_{1}+E_{2} m_{2}\right.\right. \\
& \left.+D_{2} E_{2}\left[\left(c_{1}+c_{2}\right) m_{2}+\left(c_{2}+c_{3}\right) m_{1}\right]\right\} s^{3} \\
& +\left\{1+D_{2}\left(c_{1}+c_{2}\right)+E_{2}\left(c_{1}+c_{3}\right)\right. \\
& +D_{2} E_{2}\left[c_{1}+c_{2}+c_{1} c_{3}+c_{2} c_{3}+\left(k_{1}+k_{2}\right) m_{2}\right. \\
& \left.\left.+\left(k_{2}+k_{3}\right) m_{1}\right]\right\} s^{2}+\left\{D_{2}\left(k_{1}+k_{2}\right)+E_{2}\left(k_{2}+k_{3}\right)\right. \\
& +D_{2} E_{2}\left[c_{1}\left(k_{2}+k_{3}\right)+c_{2}\left(k_{1}+k_{3}\right)\right. \\
& \left.\left.+c_{3}\left(k_{1}+k_{2}\right)\right]\right\} s+D_{2} E_{2}\left[\left(k_{1}+k_{2}\right)\left(k_{2}+k_{3}\right)-k_{2}^{2}\right] .
\end{aligned}
$$

Next note that since $w$ and $y_{x}$ are colocated, $u$ and $z_{x}$ are colocated, and $z_{x}$ and $y_{x}$ are velocities, it follows that $G_{z_{x} u}(s)$ and $G_{y_{x} w}(s)$ are positive real and hence minimum phase, and thus $A-B E_{2}^{-1} E_{1}$ and $A-D_{1} D_{2}^{-1} C$ are asymptotically stable for all $E_{2}, D_{2}>0$. Therefore, it follows from Theorem 6.1 that the approximate zero spillover controller stabilizes the closed-loop system. Finally, since $\lim _{E_{2}, D_{2} \rightarrow 0} g(s)=1$, it follows that:

$$
\lim _{E_{2}, D_{2} \rightarrow 0} G_{\mathrm{AZSC}}(s)=-c_{2}-\frac{k_{2}}{s}=G_{\mathrm{ZSC}}(s)
$$

which verifies (22). It can be seen that the loop transfer function $L(s)$ has four poles due to the cancellation of a pole-zero pair at the origin.

$$
\begin{aligned}
{\left[\begin{array}{l}
z_{x} \\
y_{x}
\end{array}\right]=\frac{\left[\begin{array}{cc}
c_{2} s^{2}+k_{2} s & m_{1} s^{3}+\left(c_{1}+c_{2}\right) s^{2}+\left(k_{1}+k_{2}\right) s \\
m_{2} s^{3}+\left(c_{2}+c_{3}\right) s^{2}+\left(k_{2}+k_{3}\right) s & c_{2} s^{2}+k_{2} s
\end{array}\right]}{\left[m_{1} s^{2}+\left(c_{1}+c_{2}\right) s+k_{1}+k_{2}\right]\left[m_{2} s^{2}+\left(c_{2}+c_{3}\right) s+k_{2}+k_{3}\right]-\left(c_{2} s+k_{2}\right)^{2}}\left[\begin{array}{l}
w \\
u
\end{array}\right] } \\
L(s)=\frac{-\left(c_{2} s+k_{2}\right)^{2}}{\left[m_{1} s^{2}+\left(c_{1}+c_{2}\right) s+k_{1}+k_{2}\right]\left[m_{2} s^{2}+\left(c_{2}+c_{3}\right) s+k_{2}+k_{3}\right]-\left(c_{2} s+k_{2}\right)^{2}} \\
S(s)=1-\frac{\left(c_{2} s+k_{2}\right)^{2}}{\left[m_{1} s^{2}+\left(c_{1}+c_{2}\right) s+k_{1}+k_{2}\right]\left[m_{2} s^{2}+\left(c_{2}+c_{3}\right) s+k_{2}+k_{3}\right]}
\end{aligned}
$$




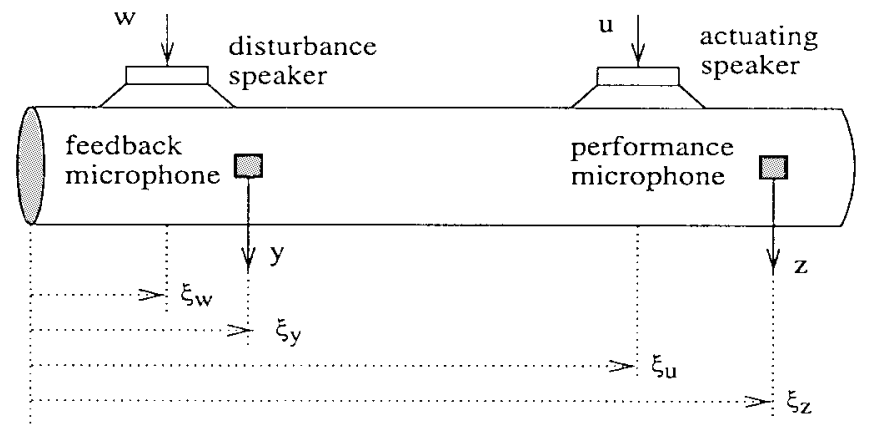

Fig. 2. Acoustic duct.

\section{B. Acoustic Duct Example}

The equations of motion for the acoustic duct shown in Fig. 2 are given by [4]

$$
\begin{aligned}
\frac{1}{c^{2}} p_{t t}(\xi, t)= & p_{\xi \xi}(\xi, t)+\rho_{0} \dot{v}_{u}(t) \delta\left(\xi-\xi_{u}\right) \\
& +\rho_{0} \dot{v}_{w}(t) \delta\left(\xi-\xi_{w}\right) \\
z_{x}(t)= & p\left(\xi_{z}, t\right), \quad y_{x}(t)=p\left(\xi_{y}, t\right)
\end{aligned}
$$

where $p(\xi, t)$ is the acoustic pressure, $c$ is the phase speed of the acoustic wave $\left(343 \mathrm{~m} / \mathrm{s}\right.$ in air at room conditions), $v_{u}(t)$ and $v_{w}(t)$ are the speaker cone velocities $(\mathrm{m} / \mathrm{s})$ of the actuating speaker and the disturbance speaker, respectively, and $\rho_{0}$ is the equilibrium density of air $\left(1.21 \mathrm{~kg} / \mathrm{m}^{3}\right.$ at room conditions). By using separation of variables, retaining $r$ modal frequencies, $p(\xi, t)$ is given by

$$
p(\xi, t)=\sum_{i=0}^{r} q_{i}(t) V_{i}(\xi) .
$$

By introducing proportional damping, the state-space realization of the system is given by (5)-(7), where

$$
\begin{aligned}
& x(t)=\left[\begin{array}{lllll}
\int_{0}^{t} q_{1}(\sigma) \mathrm{d} \sigma & q_{1}(t) & \cdots & \int_{0}^{t} q_{r}(\sigma) \mathrm{d} \sigma & q_{r}(t)
\end{array}\right]^{\mathrm{T}} \\
& u(t) \triangleq A_{\mathrm{s}} v_{u}(t), \quad w(t) \triangleq A_{\mathrm{s}} v_{w}(t) \\
& A \triangleq\left[\begin{array}{ccccc}
0 & 1 & & & \\
-\omega_{\mathrm{n} 1}^{2} & -2 \zeta_{1} \omega_{\mathrm{n} 1} & & & \\
& & \ddots & & \\
& & & 0 & 1 \\
& & & -\omega_{\mathrm{n} r}^{2} & -2 \zeta_{r} \omega_{\mathrm{n} r}
\end{array}\right]
\end{aligned}
$$

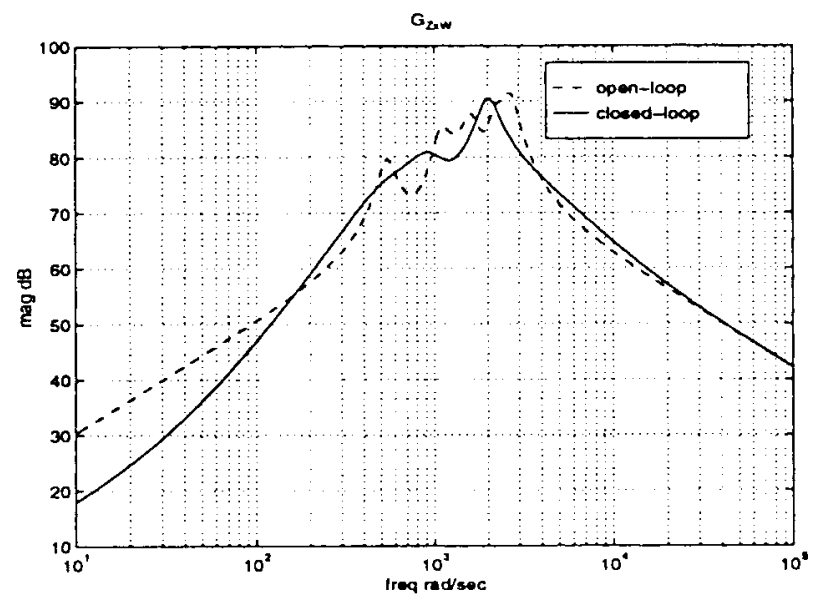

(a)

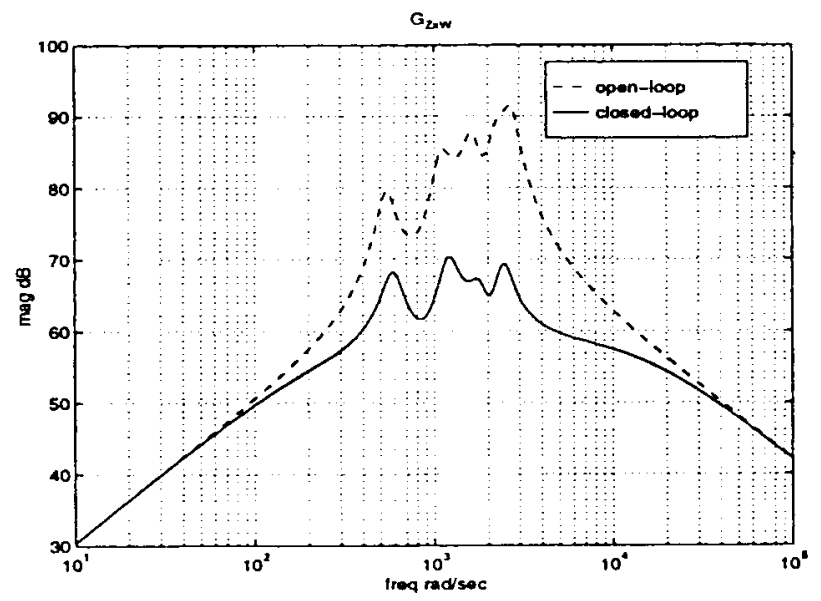

(b)

Fig. 3. (a) The open-loop and closed-loop transfer functions from $w$ to $z_{x}$ with colocated measurement and performance and with colocated control and disturbance indicate spillover. (b) The open-loop and closed-loop transfer functions from $w$ to $z_{x}$ with noncolocated measurement and performance and with noncolocated control and disturbance indicate the absence of spillover.

$$
B \triangleq\left[\begin{array}{c}
0 \\
\frac{\rho_{0}}{A_{\mathrm{s}}} V_{1}\left(\xi_{u}\right) \\
\vdots \\
0 \\
\frac{\rho_{0}}{A_{\mathrm{s}}} V_{r}\left(\xi_{u}\right)
\end{array}\right], \quad D_{1} \triangleq\left[\begin{array}{c}
0 \\
\frac{\rho_{0}}{A_{\mathrm{s}}} V_{1}\left(\xi_{w}\right) \\
\vdots \\
0 \\
\frac{\rho_{0}}{A_{\mathrm{s}}} V_{r}\left(\xi_{w}\right)
\end{array}\right]
$$

$$
G_{\mathrm{AZSC}}(s) \sim\left[\begin{array}{cccc|c}
0 & 1 & 0 & 0 & 0 \\
-\frac{k_{1}+k_{2}}{m_{1}} & -\frac{c_{1}+c_{2}}{m_{1}}-\frac{1}{m_{1} D_{2}} & \frac{k_{2}}{m_{1}} & \frac{c_{2}}{m_{1}} & \frac{1}{m_{1} D_{2}} \\
0 & 0 & 0 & 1 & 0 \\
\frac{k_{2}}{m_{2}} & \frac{c_{2}}{m_{2}} & -\frac{k_{2}+k_{3}}{m_{2}} & -\frac{c_{2}+c_{3}}{m_{2}}-\frac{1}{m_{2} E_{2}} & 0 \\
\hline 0 & 0 & 0 & -\frac{1}{E_{2}} & 0
\end{array}\right]=\frac{-c_{2}-\frac{k_{2}}{s}}{g(s)}
$$




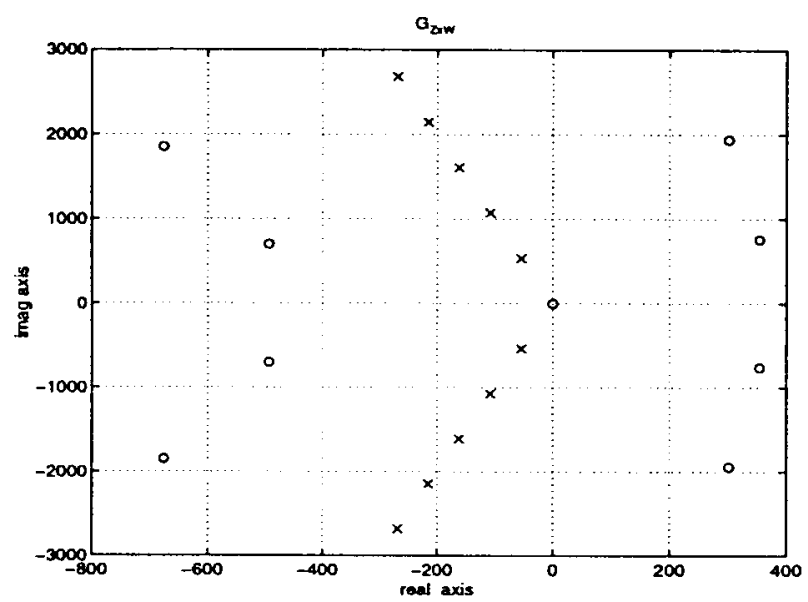

(a)

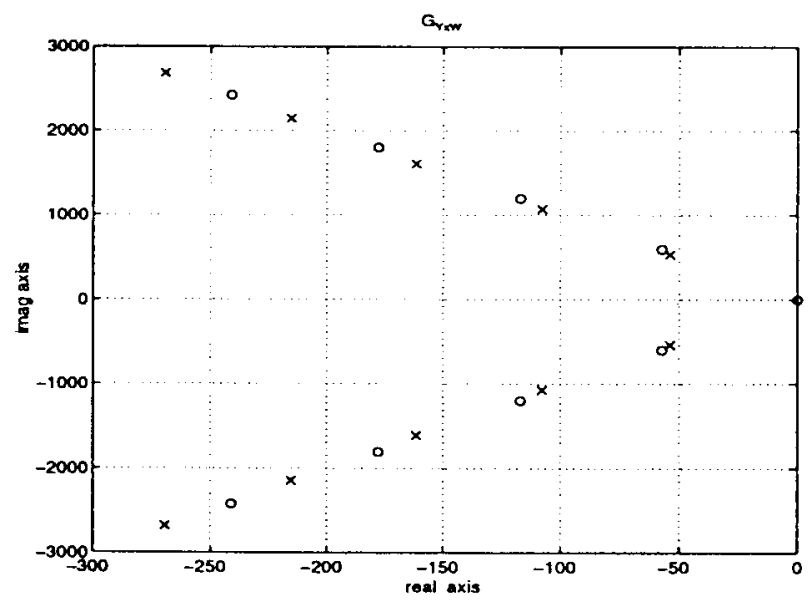

(c)

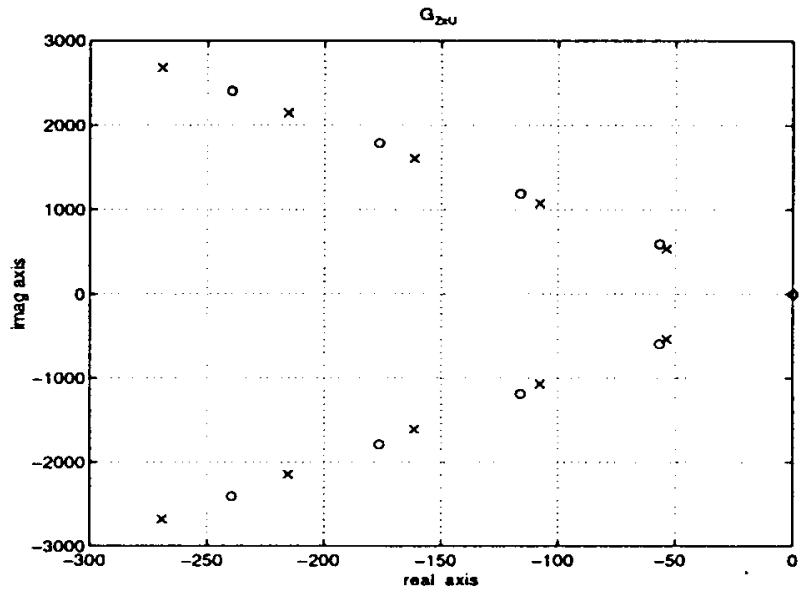

(b)

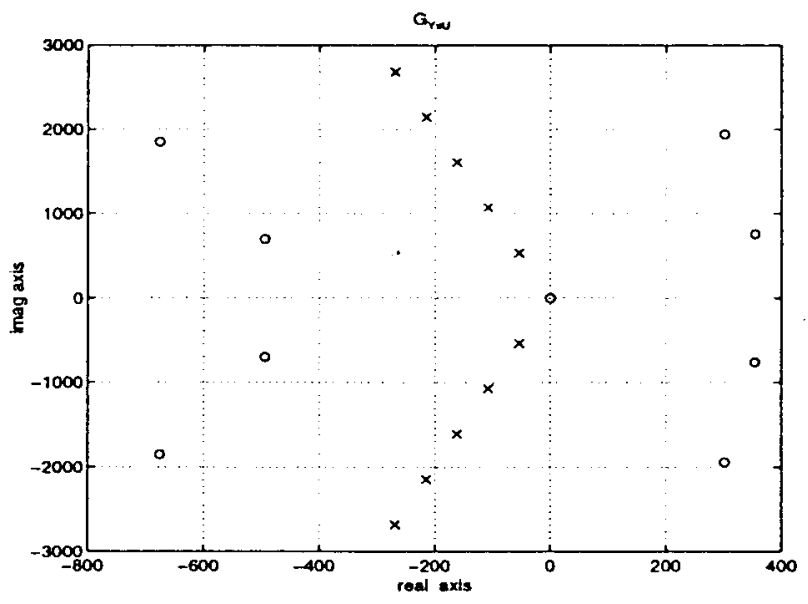

(d)

Fig. 4. Pole-zero plots of the transfer functions (a) $G_{z_{z} w}(s)$, (b) $G_{z_{x} u}(s)$, (c) $G_{y_{x^{x}} w}(s)$, and (d) $G_{y_{x} u}(s)$ with noncolocated measurement and performance and with noncolocated control and disturbance show that $G_{z_{x}} u(s)$ and $G_{y_{x} w}(s)$ satisfy pole-zero interlacing and thus are minimum phase.

$$
\begin{aligned}
C & \triangleq\left[\begin{array}{lllll}
0 & V_{1}\left(\xi_{y}\right) & \cdots & 0 & V_{r}\left(\xi_{y}\right)
\end{array}\right] \\
E_{1} & \triangleq\left[\begin{array}{lllll}
0 & V_{1}\left(\xi_{z}\right) & \cdots & 0 & V_{r}\left(\xi_{z}\right)
\end{array}\right]
\end{aligned}
$$

and

$$
\omega_{\mathrm{n} i} \triangleq \frac{i \pi}{L} c, \quad V_{i}(\xi) \triangleq c \sqrt{\frac{2}{L}} \sin \frac{i \pi}{L} \xi, \quad i=1,2, \cdots, r
$$

where $A_{\mathrm{s}}$ is the cross-sectional area of the speaker, $\zeta_{i}$ is the damping ratio of the $i$ th acoustic mode, and $L$ is the length of the duct. For simplicity, speaker dynamics [4] are neglected. Numerical values for this example are $A_{s}=0.0025 \mathrm{~m}^{2}, L=2$ $\mathrm{m}, \zeta_{i}=0.1, i=1, \cdots, 5, \xi_{w}=0.121 \mathrm{~m}$, and $\xi_{z}=1.937 \mathrm{~m}$.

In Sections III and IV it was shown that if either the state performance variable $z_{x}$ and the measurement $y_{x}$ are colocated or the control input $u$ and the disturbance signal $w$ are colocated, then spillover cannot be prevented. This property is illustrated in Fig. 3(a) by applying LQG with $\xi_{y}=\xi_{z}$, $\xi_{u}=\xi_{w}, D_{2}=0.1$, and $E_{2}=2000$. Note that since $\xi_{z}=\xi_{y}$ and $\xi_{w}=\xi_{u}, G_{\mathrm{ZSC}}(s)$ does not exist for this case. In contrast, consider the case in which the performance microphone and the measurement microphone are noncolocated and the control speaker and the disturbance signal are noncolocated. In this case, by letting $\xi_{y}=\xi_{w}$ and $\xi_{u}=\xi_{z}$ and applying LQG with
$D_{2}=0.1$ and $E_{2}=2000$, it can be seen in Fig. 3(b) that spillover has been avoided.

Next, we apply Theorem 6.1 with $\xi_{y}=\xi_{w}, \xi_{u}=\xi_{z}$, and a decreasing sequence of values of $D_{2}>0$ and $E_{2}>0$, where $D_{2}$ is chosen to be equal to $E_{2}$. As can be seen from Fig. 4(b) and (c), the transfer function $G_{y_{x} w}(s)$ from the disturbance speaker to the measurement microphone and the transfer function $G_{z_{x} u}(s)$ from the control speaker to the performance microphone are both minimum phase. ${ }^{4}$ Thus, in accordance with Proposition 4.1, it is verified numerically that $G_{\mathrm{ZSC}}(s)$ stabilizes the sensitivity function $S(s)$. Note from Fig. 4(d), however, that the transfer function $G_{y_{x} u}(s)$ from the control speaker to the measurement microphone is nonminimum phase.

Fig. 5(b) shows the magnitude of the sensitivity function $S(s)$ with $G_{\mathrm{C}}(s)=G_{\mathrm{AZSC}}(s)$ as $D_{2}$ and $E_{2}$ decrease. The poor form of the sensitivity is consistent with the fact that the transfer function from the control speaker to the measurement microphone is nonminimum phase, and thus the system is inherently difficult to control [20]. Note that the controller,

\footnotetext{
${ }^{4}$ In fact, these colocated impedance transfer functions are positive real since the input is volume velocity, the output is pressure, and their product has the dimensions of power.
} 


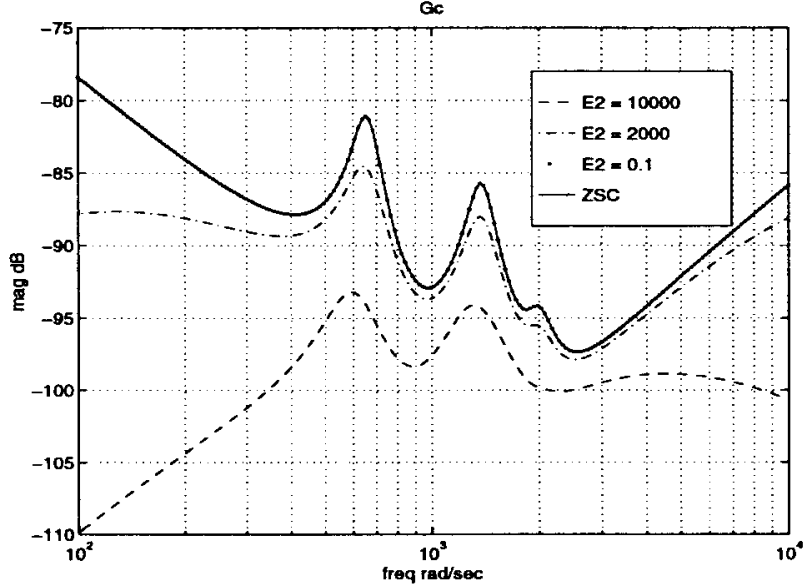

(a)

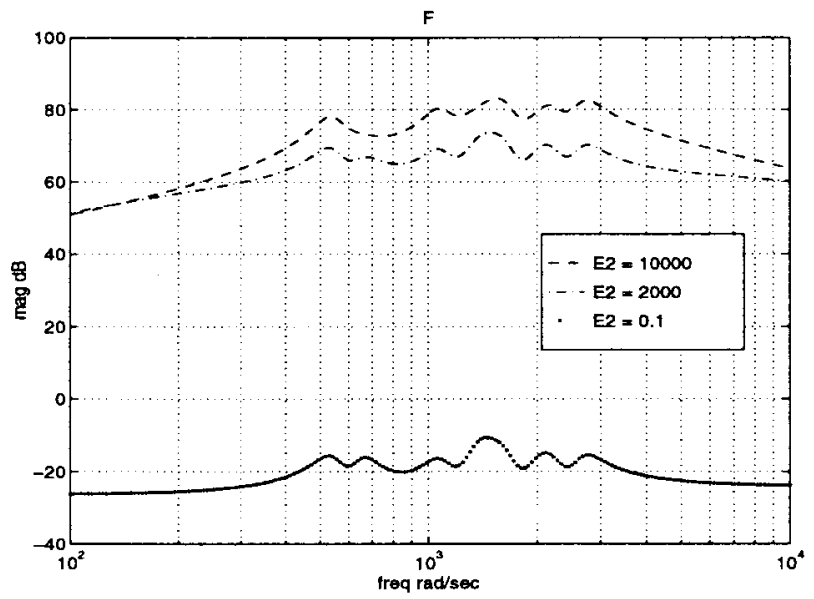

(c)

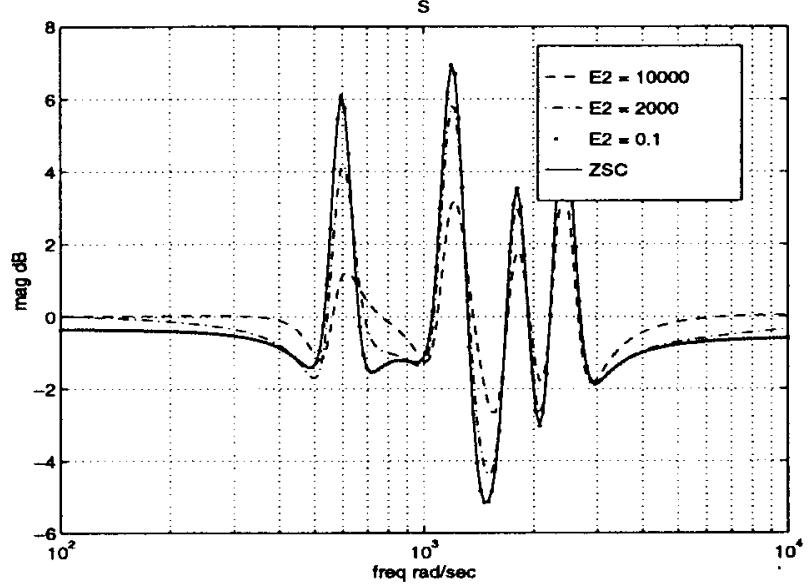

(b)

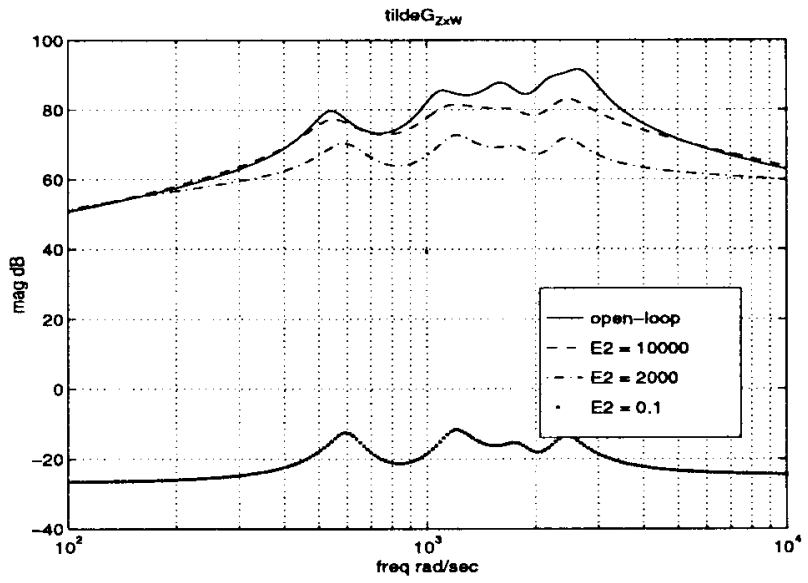

(d)

Fig. 5. For the case of noncolocated measurement and performance and noncolocated control and disturbance: (a) The approximate zero spillover controller $G_{\mathrm{AZSC}}(s)$ is relatively insensitive to $D_{2}$ and $E_{2}$. (b) The sensitivity function $S(s)$ with $G_{\mathrm{c}}(s)=G_{\mathrm{AZSC}}(s)$ is relatively insensitive to $D_{2}$ and $E_{2}$. (c) The spillover function $F\left[G_{\mathrm{C}}(s)\right]$ is highly sensitive to $G_{\mathrm{C}}(s)$, so that small changes in $G_{\mathrm{AZSC}}(s)$ due to decreasing $D_{2}$ and $E_{2}$ cause large suppression of $F\left[G_{\mathrm{AZSC}}(s)\right]$. (d) The magnitude of the closed-loop transfer function $\tilde{G}_{z_{x} w}(s)$ with the controller $G_{\mathrm{AZSC}}(s)=G_{\mathrm{c}, \text { opt }}(s)$ decreases as $D_{2}$ and $E_{2} \rightarrow 0$, which indicates that the $-\infty \mathrm{dB}$ performance of the zero spillover controller $G_{\mathrm{ZSC}}(s)$ is recovered from a sequence of approximate zero spillover controllers.

and thus the sensitivity function, does not change significantly as $D_{2}$ and $E_{2}$ decrease as shown in Fig. 5(a) and (b). However, the magnitude of $F\left[G_{\mathrm{AZSC}}(s)\right]$ approaches $-\infty \mathrm{dB}$ as $D_{2} \rightarrow 0$ and $E_{2} \rightarrow 0$ as shown in Fig. 5(c). Hence the magnitude of the closed-loop transfer function with the controller $G_{\mathrm{AZSC}}(s)=$ $G_{c, \text { opt }}(s)$ decreases as $D_{2} \rightarrow 0$ and $E_{2} \rightarrow 0$ as shown in Fig. 5(d). The numerical results indicate that the sequence of approximate zero spillover controllers $G_{\mathrm{AZSC}}(s)$ recovers the $-\infty \mathrm{dB}$ performance of the zero spillover controller $G_{\mathrm{ZSC}}(s)$.

\section{CONCLUSIONS}

In this paper, spillover was analyzed in terms of the Bode integral constraint, and it was shown that the feedforward controller given in [10] can be viewed as a zero spillover controller in a feedback formulation. For implementation purposes, a realization of the approximate zero spillover controller was given. For analytical and numerical examples, the zero spillover controller was shown to be a limiting case of an $\mathrm{H}_{2}$-optimal controller. This property suggests that the zero spillover controller is the solution to a singular LQG prob- lem. Current research is focusing on this problem using the techniques of [15] and [25]-[27].

\section{ACKNOWLEDGMENT}

The authors thank S. Bhat for providing the example of Section VII-A and C. V. Hollot, University of Massachusetts, Amherst, for suggesting Proposition 4.1.

\section{REFERENCES}

[1] S. M. Kuo and D. R. Morgan, Active Noise Control Systems. New York: Wiley-Interscience, 1996.

[2] A. J. Hull, C. J. Radcliffe, and S. C. Southward, "Global active noise control of a one-dimensional acoustic duct using a feedback controller," J. Dynamic Syst. Measurement, Contr., vol. 115, pp. 488-494, 1993.

[3] X. H. Yang, J. van Niekerk, K. S. Parwani, A. Packard, and J. Tingue, "Attenuation of structurally generated interior noise through active control," in Proc. Amer. Contr. Conf., San Francisco, CA, 1993, pp. $1-7$.

[4] J. Hong, J. C. Akers, R. Venugopal, M. N. Lee, A. G. Sparks, P. D. Washabaugh, and D. S. Bernstein, "Modeling, identification, and feedback control of noise in an acoustic duct," IEEE Trans. Contr. Syst. Technol., vol. 4, pp. 283-291, 1996. 
[5] D. G. MacMartin, "A feedback perspective on the LMS disturbance feedforward algorithm," in Proc. Amer. Contr. Conf., Baltimore, MD, June 1994, pp. 1632-1636.

[6] H. Bode, Network Analysis and Feedback Amplifier Design. New York: Van Nostrand Reinhold, 1945.

[7] I. M. Horowitz, Synthesis of Feedback Systems. New York: Academic, 1963.

[8] J. S. Freudenberg and D. P. Looze, "Right half-plane poles and zeros and design tradeoffs in feedback systems," IEEE Trans. Automat. Contr., vol. AC-30, pp. 555-565, 1985.

[9] R. H. Middleton, "Tradeoffs in linear control system design," Automatica, vol. 27, pp. 281-292, 1991 .

[10] A. Roure, "Self-adaptive broadband active sound control system," $J$. Sound Vibration, vol. 101, pp. 429-441, 1985.

[11] P. A. Nelson and S. J. Elliott, Active Control of Sound. London: Academic, 1992.

[12] D. MacMartin and J. How, "Implementation and prevention of unstable optimal compensators," in Proc. Amer. Contr. Conf., Baltimore, MD, June 1994, pp. 2190-2195.

[13] M. J. Balas, "Trends in large space structures control theory: Fondest hopes, wildest dreams," IEEE Trans. Automat. Contr., vol. AC-27, pp. 522-535, 1982

[14] D. C. Hyland, J. L. Junkins, and R. W. Longman, "Active control technology for large space structures," J. Guid. Contr. Dynamics, vol. 16, pp. 801-821, 1993.

[15] H. Kwakernaak and R. Sivan, "The maximally achievable accuracy of linear optimal regulators and linear optimal filters," IEEE Trans. Automat. Contr., vol. AC-17, pp. 79-86, 1972.

[16] R. H. Cannon, Jr. and D. E. Rosenthal, "Experiments in control of flexible structures with noncolocated sensors and actuators," $J$.
Guidance, vol. 7, pp. 546-553, 1984.

[17] D. G. MacMartin, "Collocated structural control for reduction of aircraft cabin noise," J. Sound Vibration, vol. 190, pp. 105-119, 1996.

[18] R. L. Clark, "Moving away from colocated control," J. Sound Vibration, vol. 190, pp. 129-136, 1996.

[19] _ "Accounting for out-of-bandwidth modes in the assumed modes approach: Implications on colocated output feedback control," $J$. Dynamic Syst. Measurement Contr., to be published.

[20] B. Francis and G. Zames, "On $H^{\infty}$ optimal sensitivity theory for SISO feedback systems," IEEE Trans. Automat. Contr., vol. AC-29, pp. 9-16, 1984.

[21] H. K. Wimmer, "Monotonicity of maximal solutions of algebraic Riccati equations,” Syst. Contr. Lett., vol. 5, pp. 317-319, 1985.

[22] S. M. Joshi and S. Gupta, "On a class of marginally stable positive real systems," IEEE Trans. Automat. Contr., vol. 41, pp. 152-155, 1996.

[23] R. Lozano-Leal and S. M. Joshi, "On the design of dissipative LQGtype controllers," in Proc. Conf. Decision Contr., Austin, TX, 1988, pp. 1645-1646.

[24] W. M. Haddad, D. S. Bernstein, and Y. W. Wang, "Dissipative $\mathrm{H}_{2} / \mathrm{H}_{\infty}$ controller synthesis," IEEE Trans. Automat. Contr., vol. 39, pp. 827-831, 1994.

[25] B. Francis, "The optimal linear-quadratic time-invariant regulator with cheap control," IEEE Trans. Automat. Contr., vol. AC-24, pp. 616-621, 1979 .

[26] Y. Halevi and Z. J. Palmor, "Extended limiting forms of optimum observers and LQG regulators," Int. J. Contr., vol. 43, pp. 193-212, 1986.

[27] Y. Halevi, W. M. Haddad, and D. S. Bernstein, "A Riccati equation approach to the singular LQG problem," Automatica, vol. 29, pp. 773-778, 1993. 